\title{
Assessment of stallion semen morphology using two different staining methods, microscopic techniques, and sample sizes
}

\author{
Katarzyna Łącka, Stanisław Kondracki, \\ Maria Iwanina, Anna Wysokińska \\ Department of Animal Reproduction and Hygiene, \\ Siedlce University of Natural Sciences and Humanities, 08-110 Siedlce, Poland \\ sk@uph.edu.pl
}

Received: September 18, 2015 Accepted: February 3, 2016

\begin{abstract}
Introduction: The aim of this study was to propose the optimal methodology for stallion semen morphology analysis while taking into consideration the staining method, the microscopic techniques, and the workload generated by a number of samples. Material and Methods: Ejaculates from eight pure-bred Arabian horses were tested microscopically for the incidence of morphological defects in the spermatozoa. Two different staining methods (eosin-nigrosin and eosin-gentian dye), two different techniques of microscopic analysis (1000× and 400× magnifications), and two sample sizes (200 and 500 spermatozoa) were used. Results: Well-formed spermatozoa and those with major and minor defects according to Blom's classification were identified. The applied staining methods gave similar results and could be used in stallion sperm morphology analysis. However, the eosin-nigrosin method was more recommendable, because it allowed to limit the number of visible artefacts without hindering the identification of protoplasm drops and enables the differentiation of living and dead spermatozoa. Conclusion: The applied microscopic techniques proved to be equally efficacious. Therefore, it is practically possible to opt for the simpler and faster 400x technique of analysing sperm morphology to examine stallion semen. We also found that the number of spermatozoa clearly affects the results of sperm morphology evaluation. Reducing the number of spermatozoa from 500 to 200 causes a decrease in the percentage of spermatozoa identified as normal and an increase in the percentage of spermatozoa determined as morphologically defective.
\end{abstract}

Keywords: stallion, semen, staining methods, spermatozoa morphology.

\section{Introduction}

Progress in breeding and insemination methods has contributed to a significant reduction in the number of males used for reproduction. Thus, the quality of ejaculates obtained from insemination males has become particularly important. Ejaculate quality is influenced by genetic factors, including the breed (31), as well as the health status, age, and subsistence conditions of the male $(3,28)$. Ejaculate parameters are also affected by the season of the year, ambient temperature and daylight duration, semen collection frequency, and other factors $(18,30)$. The ejaculates of individual animals differ in physical traits and in the incidence of morphological defects in the spermatozoa (19).

Each collected ejaculate is analysed to determine the basic physical traits, such as ejaculate volume, number and motility of spermatozoa, and sperm concentration. Sperm morphology is also subject to assessment. This analysis is of strategic importance (20). Sperm morphology is considered to be one of the most important indices of potential male fertility (8). The structure and sizes of spermatozoa influence their correct functioning, including their capability of acrosomal reaction (23) and bonding with the pellucid zone of the oocyte (12), thus affecting the potential fertility of the male (24). Sperm morphology assessment consists of determining the percentage of spermatozoa with defective morphological structure and the share of spermatozoa with major or minor defects (25). Male fertility has been shown to depend on the incidence of morphologically defective spermatozoa (1). Even a slight increase in the percentage of spermatozoa with major defects can lower male fertility (7). It was 
observed that male fertility clearly depends on the percentage of spermatozoa with morphological defects (14). The relationship is inversely proportional, which means that the more spermatozoa with structural defects, the lower the insemination efficacy.

Sperm classification systems tend to be modified and adapted for various species of animals. The classical system, developed by Blom (4) for bull spermatozoa, was adjusted for the assessment of stallion sperms (16). The system divides spermatozoa into normal ones, those with primary defects, and those with secondary defects. Major sperm defects usually stem from anomalies during spermatogenesis. Minor defects, in turn, arise later on, in semen outlet ducts. Minor sperm defects can also be caused outside the body of the male, e.g. during laboratory procedures or microscopic samples preparation (26). Excessively long storage or incorrect storage temperature can also lead to minor defects of spermatozoa (21).

Determination of changes in the morphological structure of spermatozoa is an important element of reproduction aptitude assessment of stallions (15). In the case of semen evaluation carried out to predict male fertility, it is important to identify the percentage share of morphologically defective spermatozoa (7). Sperm morphology is evaluated in microscopic analyses. Such examinations require fresh semen for microscopic preparations, which are fixed and stained. Staining is a physicochemical process in which the dye penetrates cellular structure. Staining allows to visualise the shapes and sizes of spermatozoa. Basically, the dye should not change the size and shape of spermatozoa, nor hide their morphological defects. There are many methods of staining microscopic preparations. It has also been shown that the staining method can affect the result of sperm morphology investigation (22).

The result of semen analysis can be influenced by the applied diagnostic technique (9), tools, and measurement accuracy. Actual magnification and number of spermatozoa under analysis are also important factors in microscopic sperm morphology assessment. Sperm morphology investigations are usually performed at, preferably, high magnifications, using immersion lenses. Such analyses should involve 500 spermatozoa (29), which require a high workload. Therefore, it is a common practice to perform this analysis on lower numbers of spermatozoa - approximately 200 (10). Maree et al. (22) assumed 100 spermatozoa as the minimal number that enables human sperm evaluation. However, the minimal sperm count indispensable for an effective assessment depends on the staining method and the type of dyes used (11).

Practical sperm morphology analyses require feasible and economic methods for staining microscopic preparations, as well as simple microscopic techniques, without the need for large magnifications and expensive and delicate equipment. The present work constitutes an attempt at assessing the efficiency of stallion sperm morphology analyses using two cheap and easily applicable methods of staining, two different microscopic techniques, and less (200 spermatozoa) or more numerous (500 spermatozoa) samples. Therefore, the aim of this study was to propose the optimal methodology for stallion semen morphology analysis while taking into consideration the staining method, the microscopic techniques, and the workload generated by a number of samples.

\section{Material and Methods}

Microscopic examination. A total of 72 microscopic preparations made from the semen of eight pure-bred Arabian horses were examined. The preparations were created using nine ejaculates. All ejaculates were microscopically tested for the incidence of morphological defects in the spermatozoa, using semen sample preparations. Both well-formed spermatozoa and those with major or minor defects according to Blom's (5) classification were identified. The incidence of morphological defects in the spermatozoa was determined on the basis of microscopic analyses of preparations made from the semen samples collected from each stallion. All analyses were conducted by the same person. Each ejaculate sample provided four preparations for morphological examination. The preparations were stained according to two methods. Two preparations were stained with eosinnigrosin (Fig. 1), and the other two with eosin-gentian dye (Fig. 2). All preparations were created and stained directly after an ejaculate was extracted.

Eosin-nigrosin staining method (differential staining). The preparations for analyses were made according to the following methodology: a drop of semen was placed on a slide preheated to $40^{\circ} \mathrm{C}$ and mixed with one part of $5 \%$ bluish eosin solution (Carl Roth Gmbh + Co. KG, Germany) and four parts of $10 \%$ nigrosin aqueous solution (Sigma-Aldrich). The samples were dried at room temperature. Two hundred of spermatozoa were assessed in each preparation. The spermatozoa were classified as those with a cell membrane structure unstained (living) and those with a damaged membrane structure stained pink (dead).

Eosin-gentian staining method. Thin, fat-free semen smears, heated up to $36^{\circ} \mathrm{C}$, were prepared. After drying, the smear was fixed in $96 \%$ ethanol. Then, it was rinsed in water and counterstained with $10 \%$ blue eosin solution for 20-60 s. The slides were rinsed in water again, and coloured for 3-5 min in gentian pigment.

Microscopic analysis. The preparations were analysed with two different microscopic techniques, using a NIKON E50i microscope with a glass magnification level $10 \times$ : 1) analysis with immersion lenses at a $1000 \times$ magnification, 2) analysis with lenses set at a $400 \times$ magnification. 
Table 1. The number of microscopic preparations stained with different methods and analysed with different microscopic techniques

\begin{tabular}{|c|c|c|c|c|c|}
\hline \multirow{2}{*}{ Staining method } & \multicolumn{4}{|c|}{ Microscopic analysis technique } & \multirow[t]{2}{*}{ Total } \\
\hline & \multicolumn{2}{|c|}{ Magnification lenses $-400 \mathrm{x}$} & \multicolumn{2}{|c|}{ Magnification lenses - 1000x } & \\
\hline Eosin-nigrosin & 9 & 9 & 9 & 9 & 36 \\
\hline Total & 18 & 18 & 18 & 18 & 72 \\
\hline
\end{tabular}

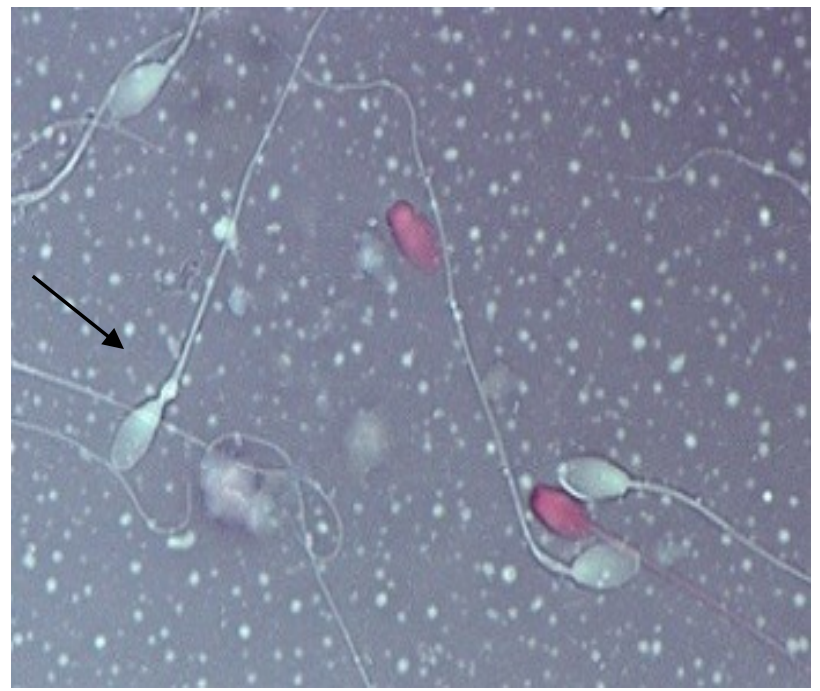

Fig. 1. A stallion semen smear stained with eosin-nigrosin method; the arrow points to the spermatozoon with the closer (proximal) protoplasmatic drop on its midpiece. The heads of spermatozoa stained pink are dead spermatozoa

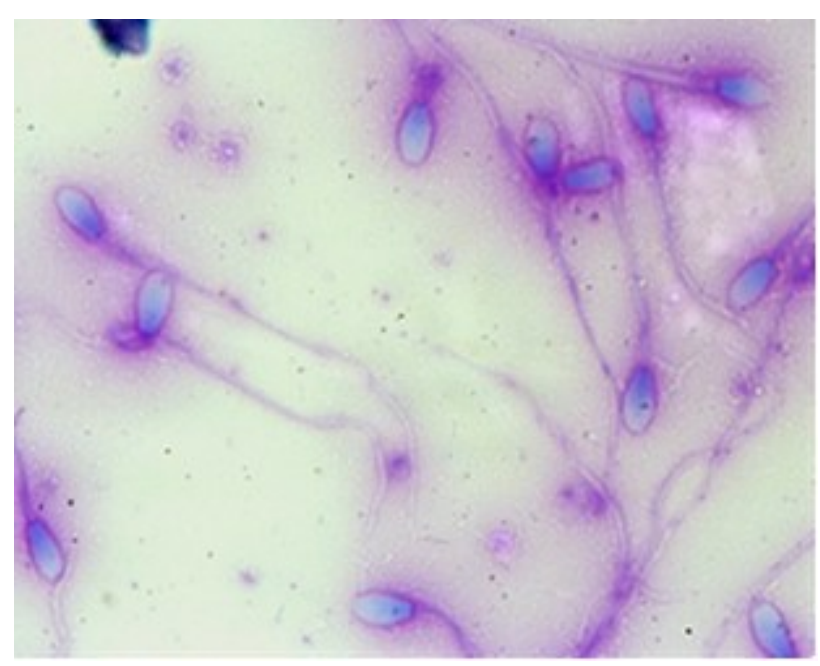

Fig. 2. A stallion semen smear stained with eosin-gentian dye

Moreover, the relationship between the precision of measuring morphological sperm defect incidence and the number of spermatozoa under analysis was determined. To this end, independently of the staining method and microscopic analysis technique, the morphology of 200 and 500 spermatozoa was evaluated. Altogether, 72 microscopic preparations were assessed (Table 1).

Statistical analysis. Experimental data were analysed using Statistica 10 PL (StatSoft, Tulsa, USA). All results are expressed as mean \pm standard deviation (SD). The obtained material was statistically analysed according to the following mathematical model: $Y_{i j}=\mu+a_{i}+e_{i j}$, where: $Y_{i j}$ is the value of the analysed parameter, $\mu$ is the population mean, $a_{i}$ is the staining method (or the microscopic analysis technique or the number of respondent spermatozoa) effect, $e_{i j}$ is the error. The significance of the differences between the groups was assessed with the Tukey's test at $\mathrm{P} \leq 0.05$ and $\mathrm{P} \leq 0.01$.

\section{Results}

Table 2 contains a juxtaposition of the results of the stallion sperm morphology analyses, depending on the staining method.

The data in Table 2 show that the applied methods of staining had similar effects on the results of the stallion sperm morphology analyses. The differences between the percentages of well-formed spermatozoa determined in the preparations stained with eosin-nigrosin and in those stained with eosingentian dye were similar $(0.17 \%)$ and statistically unconfirmed. The differences between the percentages of spermatozoa with major $(0.14 \%)$ and minor defects $(0.31 \%)$ identified in the preparations stained with both methods were also negligible and statistically insignificant.

Table 3 contains a juxtaposition of the results of the stallion sperm morphology analyses, depending on the magnification.

The analysis at $400 \times$ magnification revealed $91.18 \%$ well-formed spermatozoa - $1.57 \%$ points fewer than in the analysis performed at $1000 \times$ magnification $(P \leq 0.05)$. No differences in major sperm defect incidence were identified between the applied microscopic techniques. On the other hand, slight differences were found in the percentages of minor sperm defects. Using a $1000 \times$ magnification, $1.03 \%$ points fewer spermatozoa with minor defects were identified, as opposed to the analysis at a $400 \times$ magnification $(\mathrm{P} \leq 0.05)$. 
Table 2. Identification of morphological defects of spermatozoa in the stallion ejaculates, depending on the staining method (means \pm SD)

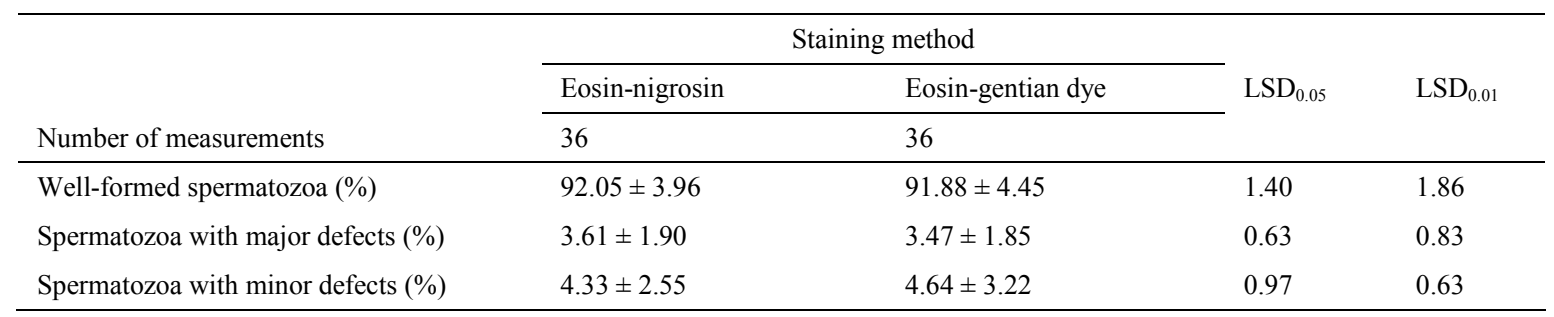

Table 3. The profile of morphological defects of spermatozoa in the stallion ejaculates in relation to the applied microscopic analysis technique (means \pm SD)

\begin{tabular}{|c|c|c|c|c|}
\hline \multirow[b]{3}{*}{ Number of measurements } & \multicolumn{2}{|c|}{ Microscopic analysis technique } & \multirow{3}{*}{$\mathrm{LSD}_{0.05}$} & \multirow{3}{*}{$\mathrm{LSD}_{0,01}$} \\
\hline & $\begin{array}{l}\text { Magnification lenses - } \\
400 \times\end{array}$ & $\begin{array}{l}\text { Magnification lenses - } \\
1000 \times\end{array}$ & & \\
\hline & 36 & 36 & & \\
\hline Well-formed spermatozoa $(\%)$ & $91.18 \pm 4.61$ & $92.75 \pm 3.60$ & 1.38 & 1.83 \\
\hline Spermatozoa with major defects (\%) & $3.80 \pm 2.09$ & $3.27 \pm 1.59$ & 0.62 & 0.82 \\
\hline
\end{tabular}

Table 4. The profile of morphological defects of the stallion sperms in relation to the numbers of analysed spermatozoa (means \pm SD)

\begin{tabular}{llll} 
& \multicolumn{2}{l}{ number of respondent spermatozoa } & \\
& 200 & 500 & LSD $_{0,05}$ \\
Number of measurements & 36 & 36 & 1.09 \\
\hline Well-formed spermatozoa (\%) & $89.36 \pm 4.14$ & $94.57 \pm 2.09$ & 1.54 \\
Spermatozoa with major defects (\%) & $4.72 \pm 1.83$ & $2.36 \pm 0.93$ & 0.49 \\
Spermatozoa with minor defects (\%) & $5.91 \pm 3.21$ & $3.06 \pm 1.57$ & 0.84 \\
\hline
\end{tabular}

Table 4 contains a juxtaposition of the results of the stallion sperm morphology analyses depending on the number of evaluated spermatozoa in the sample.

The data juxtaposed in Table 4 present the relationship between the results of the sperm morphology assessments and the numbers of analysed spermatozoa. With a higher number of spermatozoa (500 cells), relatively fewer morphological defects were identified than in the assessment of only 200 spermatozoa. In the analysis of 500 spermatozoa, $2.36 \%$ fewer sperms with major defects and $2.85 \%$ fewer sperms with minor defects were identified, as compared with the assessment of 200 spermatozoa $(\mathrm{P} \leq 0.01)$. The study of 500 spermatozoa also revealed a significantly higher (by $4.72 \%$ ) share of sperms with correct morphology $(\mathrm{P} \leq 0.01)$. On the basis of the investigations, it can be concluded that the total number of the analysed spermatozoa is significant (500 spermatozoa). In the case of a high number of spermatozoa subjected to the morphology assessment, the study results indicate higher stallion sperm quality.

\section{Discussion}

The data presented in Table 2 show that the use of the eosin-nigrosin and eosin-gentian dye staining methods gives similar results and both methods can be used to examine stallion sperm morphology. Brito et al. (6) showed that eosin-nigrosin staining method can give similar results in stallion sperm morphology analyses to those obtained with Papanicolaou staining. Similar observations are also reported by other researchers (26). The preparation staining methods can, however, significantly affect the identification of morphological defects in the spermatozoa, which is of particular importance for the automatic assessment of sperm morphology by means of the CASA system. The influence of the staining method on the results of morphological and morphometric analyses of spermatozoa has been repeatedly determined (22). Hidalgo et al. (13) revealed that the staining method (Diff-Quik, Hemacolor, and Harris' haematoxylin) affects the accuracy of image processing and identification of morphological and morphometric parameters of stallion spermatozoa. The present article compares the efficiency of stallion sperm morphology assessments using preparations stained according to two methods: eosin-nigrosin and eosin-gentian dye. We found that the use of these two methods of staining gives similar results with regard to the evaluation of the incidence of sperm morphology defects. In practice, however, these methods offer quite different possibilities. The eosin-nigrosin stain is a negative, differential dye (27) which, in comparison with other staining methods, makes it possible to limit the amount 
of visible artefacts and does not impede the identification of protoplasmatic drops. The advantage of this method is also the possibility of differentiating living and dead spermatozoa (17). In this method, spermatozoa with structurally intact cellular membranes will not be stained, whereas those with damaged cellular membranes will change their colour (2). Spermatozoa dyed red or dark pink are assumed to be dead, whereas those which have turned greenish or pale pink are considered to be alive. In contrast to staining with eosin only, the combination with nigrosin produces a dark background, which enables better visualisation of the spermatozoa. The higher percentage of stained spermatozoa, i.e. dead ones, the lower fertility of the male. In the case of staining with the eosin-gentian dye, both the living and dead spermatozoa have a purple hue. A significant aspect of both methods is the degree of semen dilution and a correct selection of dye concentration, apart from the duration of exposure to the dye (2). It is important that the structure and functions of all the morphological elements of the spermatozoon are correct. That is why morphology assessments not only refer to the defects of the head but also to those of the neck, mid-piece, and tail. The present study did not reveal any influence of the applied staining methods on the efficacy of morphological defect identification within the particular sperm elements.

We found that the use of small magnifications $(400 \times)$ revealed a slightly higher percentage of morphologically defective spermatozoa. However, the differences were negligible and were only confirmed in relation to minor morphological defects. Assuming the assessment with a higher, $1000 \times$ magnification, using immersion oil, is more accurate, it seems that the assessment with a 400× magnification slightly overestimates the results of the identification of morphologically defective spermatozoa. However, the difference is slight and, by far, does not reduce the number of identifiable morphological anomalies. It is then admissible to take advantage of the simpler and faster microscopic technique of analysing stallion sperm morphology using $400 \times$ magnification without immersion oil.

The data clearly show that the number of spermatozoa under analysis significantly influences the results of sperm morphology investigation. The differences based on the number of analysed spermatozoa were large and turned out to be highly significant. It is also worth noting that the reduction of spermatozoa to 200 leads to overestimation of the percentage of identifiable morphological defects. It seems logical that, with a smaller pool (i.e. a lower number of assessed spermatozoa), each identified defect of sperm structure considerably gains in significance. A reduction in the number of spermatozoa subjected to assessment is used in order to facilitate processing and makes it less time-consuming in the case of certain analyses (10). Maree et al. (22) found that the number of human spermatozoa is significant for the results of the assessment and suggested that at least 100 spermatozoa should be examined for the sperm population sample to remain representative. In the case of manual sperm morphology assessments, it is recommended to analyse at least 200 spermatozoa in each sample.

The use of the eosin-nigrosin and eosin-gentian dye staining methods leads to similar results in sperm morphology analyses and both methods can be used to investigate stallion sperm morphology. However, considering the fact that eosin-nigrosin staining makes it possible to limit the amount of visible artefacts and differentiate living and dead spermatozoa, without impeding the identification of protoplasmatic drops, it is highly recommendable for use in practical stallion sperm morphology examinations. The technique-related differences in the identification of morphological defects in spermatozoa turned out to be negligible. It is then admissible to take advantage of the simpler and faster microscopic technique of analysing stallion sperm morphology using $400 \times$ magnification. The number of spermatozoa subjected to analysis clearly affects the results of sperm morphology evaluation. Reduction of the number of analysed spermatozoa from 500 to 200 causes a decrease in the percentage of spermatozoa identified as normal and an increase in the percentage of spermatozoa identified as morphologically defective.

Conflict of Interests Statement: The authors declare that there is no conflict of interests regarding the publication of the article.

Financial Disclosure Statement: This study was financed from statutory activity.

Animal Rights Statement: The experiments on animals were conducted in accordance with local Ethical Committee laws and regulations as regards care and use of laboratory animals.

\section{References}

1. Alm K., Peltoniemi O., Koskinen E., Andersson M.: Porcine field fertility with two different insemination doses and the effect of sperm morphology. Reprod Domes Anim 2006, 41, 210-213.

2. Almadaly E., Farrag F., Shukry M., Murase T.: Plasma membrane integrity and morphology of frozen-thawed bull spermatozoa supplemented with desalted and lyophilized seminal plasma. Global Vet 2014, 13, 753-766.

3. Banaszewska D., Kondracki S., Wysokińska A.: Effect of age on the dimensions and shape of spermatozoa of Large White Polish boars. Arch Tierz 2011, 54, 504-514.

4. Blom E.: The ultrastructure of some characteristic sperm defect and a proposal for a new classification of the bull spermiogram. Natl Med J India 1973, 25, 383-391.

5. Blom E.: Studies on seminal vesiculitis in the bull: II. Proposal for a new classification of the spermiogram. Med Weter 1981, 37, 239-242.

6. Brito L.F., Greene L.M., Kelleman A., Knobbe M., Turner R.: Effect of method and clinical on stallion sperm morphology evaluation. Theriogenology $2011,76,745-750$. 
7. Brodzki P., Wrona Z., Klimont M., Krakowski L.: Morphological characteristics of boar spermatozoa in the annual production cycle. Med Weter 2015, 71, 109-113.

8. Coetzee K., Kruger T.F., Lombard C.J.: Predictive value of normal sperm morphology: a structured literature review. Hum. Reprod Update 1998, 4, 73-82.

9. Foster M.L., Love C.C., Varner D.D., Brinsko S.P., Hinrisch K., Teague S., LaCaze K., Blanchard T.L.: Comparison of methods for assessing integrity of equine sperm membrane. Theriogenology 2011, 76, 334-341.

10. Freneau G.E., Chenoweth P.J., Ellis R., Rupp G.: Sperm morphology of beef bulls evaluated by two different methods. Anim Reprod Sci 2010, 118, 176-181.

11. García-Herreros M., Aparicio I.M., Barón F.J., GarcíaMarín L.J., Gil M.C.: Standardization of sample preparation, staining and sampling methods for automated sperm head morphometry analysis of boar spermatozoa. Int J Andro 2006, $29,553-563$.

12. Garrett C., Liu D.Y., Baker H.W.: Selectivity of the human sperm-zona pellucida binding process to sperm head morphometry. Fertil Steril 1997, 67, 362-371.

13. Hidalgo M., Rodríguez I., Dorado Sanz J., Soler C.: Effect of sample size and staining methods on stallion sperm morphometry by the Sperm Class Analyzer. Vet Med 2005, 50, 24-32.

14. Hidalgo M., Rodríguez I., Dorado J.: Influence of staining and sampling procedures on goat sperm morphometry using the Sperm Class Analyzer. Theriogenology 2006, 66, 996-1003.

15. Holroyd R.G., Doogan V.J., De Faveri J.D., Fordyce G., McGowan M.R., Bertram J.D., Vankan D.M., Fitzpatrick L.A., Jayawardhana G.A., Miller R.G.: Bull selection and use in northern Australia 4: calf output and predictors of fertility of bulls in multiple-sire herds. Anim Reprod Sci 2002, 71, 67-79.

16. Jasko D.J., Lein D.H., Foote R.H.: Determination of the relationship between sperm morphologic classifications and fertility in stallions: 66 cases (1987-1988). J Am Vet Med Assoc 1990, 197, 389-394

17. Jasko D.J.: Evaluation of stallion semen. Vet Clin N Am Equine Pract 1992, 8, 129-148.

18. Katila T.: In vitro evaluation of frozen-thawed stallion semen: a review. Acta Vet Scand 2001, 42, 199-217.
19. Knecht D., Srodoń S., Dudziński K.: The influence of boar breed and season on semen parameters. S Afr J Anim Sci 2014, 44, $1-9$.

20. Kondracki S., Iwanina M., Wysokińska A., Huszno M.: Comparative analysis of Duroc and Pietrain boar sperm morphology. Acta Vet Brno 2012, 81, 195-199.

21. Kondracki S., Banaszewska D., Bajena M., Komorowska K. Kowalewski D.: Correlation of frequency of spermatozoa morphological alterations with sperm concentration in ejaculates of Polish Landrace boars. Acta Vet Beograd 2013, 63, 513-524.

22. Krakowski L., Piech T., Sławińska-Brych A., Tatara M.R. Bartoszek J.: Quality of Cooley semen of cold-blooded stallions evaluated with the use of apoptosis and DNA defragmentation markers. Med Weter 2014, 70, 573-578.

23. Maree L., Du Plessis S.S., Menkveld R., Van der Horst G.: Morphometric dimensions of the human sperm head depend on the staining method used. Hum Reprod 2010, 25, 1369-1382.

24. Menkveld R., El-Garem Y., Schill W.B., Henkel R.: Relationship between human sperm morphology and acrosomal function. J Assist Reprod Gen 2003, 20, 432-438.

25. Menkveld R.: Clinical significance of the low normal sperm morphology value as proposed in the $5^{\text {th }}$ WHO laboratory manual for the examination and processing of human semen. Asian J Androl 2010, 12, 47-58.

26. Menkveld R.: Sperm morphology assessment using strict (tygerberg) criteria. Methods Mol Biol 2013, 927, 39-50.

27. Nowakowska I., Pozór M.A.: Sperm morphology of stallions using four different methods. Med Weter 2003, 59, 351-354.

28. O'Connel M., McClure N., Lewis S.E.: The effect of cryopreservation on sperm morphology, motility and mitochondrial function. Hum Reprod 2002, 17, 704-709.

29. Smital J.: Effects influencing boar semen. Anim Reprod Sci 2009, 110, 335-346.

30. Wysokińska A., Kondracki S., Banaszewska D.: Morphometrical characteristics of spermatozoa in Polish Landrace boars with regard to the number of spermatozoa in an ejaculate. Reprod Biol 2009, 9, 271-282.

31. Wysokińska A., Kondracki S.: Assessment of sexual activity levels and their association with ejaculate parameters in twobreed hybrids and purebred Duroc and Pietrain boars. Ann Anim Sci 2014, 14, 559-571. 